\title{
Study on Logistics Management Practice under the Background of Intelligent Logistics
}

\begin{abstract}
Wan Yi Xiao
Jiangxi Vocational technical College of Industry \&Trade, jiangxi,nanchang,330038

739960103@qq.com

ABSTRACT

Along with our country in reform and opening-up, China's economic development is very rapid, today already has undergone earth-shaking changes, people's material life level rising, the pace of life of people more and more fast, online shopping also gradually thriving, have become an indispensable part of people life, the development of logistics industry is also very quickly, so the concept of the intelligent logistics is put forward, and the wisdom logistics is simply through the Internet information technology to the multiple link in the logistics industry operation with high efficiency, thus can greatly save the economic costs, so as to promote the development of the logistics industry. In such an era, people will have new expectations for the logistics industry, and this paper mainly focuses on the logistics management practice under the background of smart logistics, through the analysis of the problems existing in the development process of domestic smart logistics, and gives some solutions. At present, when the development of smart logistics in China is not quite complete, the research and analysis of smart logistics is very necessary, which can help promote the development of smart logistics in China. Therefore, I hope this paper can play a certain reference and inspiration role for later research.
\end{abstract}

Keywords: Intelligent logistics, Background, Logistics management, Practice research

\section{智慧物流背景下的物流管理实践研究}

\author{
肖婉宜
}

\author{
江西工业贸易职业技术学院 江西 南昌 330038 \\ 739960103@qq.com
}

\section{摘要}

随着我国改革开放在不断深入, 我国经济发展也非常迅速, 当今时代早已发生了翻天覆地的改变, 人们的生 活物质水平在不断上升, 人们的生活节奏越来越快, 线上购物也逐渐兴盛起来, 已经成为人们生活中必不可 少的一部分, 物流行业的发展也十分迅速, 于是智慧物流的概念被提出, 智慧物流简单来讲就是通过互联网 信息技术将物流行业中的多个环节进行高效率运作, 从而能够大大节省经济成本, 从而促进整个物流行业的 发展。在这样的时代背景下, 人们对于物流行业也会产生新的期待, 而本文就主要围绕智慧物流背景下的物 流管理实践进行研究和探讨, 通过对当下国内智慧物流发展过程中所存在的问题进行了剖析, 并且给出了一 定的解决对策。在我国智慧物流发展还不是十分完善的的当下, 对智慧物流的研究分析是十分必要的, 对促 进我国智慧物流的发展能够起到帮助作用, 因此希望本文能够对后期研究起到一定的参考和启发作用。

关键词: 智慧物流; 背景; 物流管理; 实践研究

\section{1. 智慧物流管理中面临的问题}

信息技术在智慧物流中能够起到非常重要的作 用, 但是就目前而言, 智慧物流管理还有待完善, 因
为其中还有很多问题没有得到解决，首先要说明，智 慧物流的基础就是运输, 通过运输, 将各个不同的行 业联系在一起，形成一种物流体系。智慧物流所涉及 的范围非常广，因此想要实施智慧物流管理是存在一 
定难度的，而和传统的物流方式相比较，可以看出两 者的不同之处, 但是无论是智慧物流管理又或是传统 物流，都存在一个共同的问题，那就是导向不明确， 因为导向不明确, 会使得智慧物流难以得到顺利的开 展, 在实际的智慧物流管理中, 会出现分工不均以及 职权混乱的问题, 而这些问题是必须要得到解决的, 因为如果不解决这些问题, 就会阻碍智慧物流的发展, 例如，上文所提到的职权混乱的问题，每个员工都应 该有其负责的岗位，而在这个岗位上出现任何问题都 应该由负责其岗位的员工进行负责，但是如果职权混 乱, 员工职责不明确, 那么就会出现越权的情况, 并 且还会使得员工缺乏工作热情, 导致物流管理的效果 难以得到保障, 虽然现阶段有很多物流企业都在提倡 改革和创新, 但是因为种种原因, 往往只能暂时搁浅, 又或是前进无力, 只有小部分的物流企业做到了改革 和创新, 但是这一小部分的物流企业中成功构建智慧 物流管理模式的也非常少, 所以无法提供更多的经验 给后来者, 小型物流企业是无法实施智慧物流的, 只 有大型物流企业能够尝试智慧物流管理模式构建。

\section{2. 解决在智慧物流背景下的物流管理问题 对策}

表 1 解决在智慧物流背景下的物流管理问题对策

\begin{tabular}{|c|c|c|c|}
\hline \multicolumn{4}{|c|}{ 解决在智慧物流背景下的物流管理问题对策 } \\
\hline $\begin{array}{c}\text { 吸纳人 } \\
\text { 才 }\end{array}$ & $\begin{array}{c}\text { 加强物流现代 } \\
\text { 化管理 }\end{array}$ & $\begin{array}{c}\text { 提高服务 } \\
\text { 质量 }\end{array}$ & $\begin{array}{c}\text { 提升物流信息 } \\
\text { 化能力 }\end{array}$ \\
\hline
\end{tabular}

\section{1. 吸纳人才}

想要推动物流企业的发展, 那么首先物流企业就 应该吸纳更多的物流专业人才, 建立专业的物流管理 团队, 因为物流管理团队的工作质量和工作效率会直 接影响到物流企业的发展, 而在吸纳物流专业人才时, 企业也需要考虑到物流专业人才是否具备先进的物 流管理理念, 又是否能够将客户的需求放在第一位, 因为只有这样的物流专业人才才能够使物流管理得 到更好的实施。

\section{2. 加强物流现代化管理}

对于物流管理人员而言, 跟上时代的脚步, 接受 更多先进的管理理念是非常有必要的, 而同时物流管 理人员也应该明确物流管理的经营理念, 推动企业的 发展, 使智慧物流的作用能够得到完全的发挥, 也帮 助企业实现利益最大化, 让企业在市场中能够博得一 席之地。

\section{3. 提高服务质量}

消费者在物流行业中所占据的地位是非常重要 的地位, 因为有了消费者, 才有了物流行业的产生, 所以物流行业的工作人员也必须尊重消费者，提升自 己的服务态度和服务质量, 低效和低素质的物流员工 会阻碍企业的发展, 也会削减企业的市场竞争力, 而 如果企业难以获得大量的物流专业人才, 企业就可以 先对企业内部的物流员工进行定期或不定期的培训, 将企业内部的物流员工打造为企业所需要的那种物 流人才。

\section{4. 提升物流信息化能力}

小型物流企业如果想要转型为大型物流企业, 那 么就要注意到物流信息化对于物流企业运营的影响, 在物流企业中, 所有的物流员工都应该提升自己的信 息技术应用能力，并且有一定的物流信息化意识，而 物流企业也应该为实现物流信息化，对物流业务流程 进行改善，同时企业还应该收集更多的市场信息，按 照用户的体验感受对物流流程进行改进。这一点可以 通过手机中的软件来对客户的信息进行收集，从而改 善用户体验。

\section{5. 对智慧物流信息化平台进行构建}

我国当下的物流行业发展中还存在着不少问题, 国家也对物流产业的发展制定了一些相关政策进行 支持，这些政策的制定出台结合了当下物流行业的发 展现状, 同时对这些问题产生的原因进行了分析, 在 此基础上制定了相应的解决方案, 出台政策。在智慧 物流信息化平台的建设的过程中，国家方面要将当下 的信息化技术进行充分利用，同时通过信息技术的优 势将其他行业进行融合发展, 共同发展进步, 互相学 习各自优势, 以达到对智慧物流信息平台的构建作用, 这样也能大大减少成本, 也可以减轻风险的发生, 物 流企业的核心竞争力也能够有所提升, 聚集各个行业 的优势。对智慧物流信息化平台的构建中也可以通过 对经营管理平台进行统一性的管理, 从而能够加强各 个企业间的链接, 能够对相关资源进行充分利用, 将 各方优势发挥到对智慧物流信息化平台的构建中去。

\section{3. 加强智慧物流背景下的高校物流管理人 才培养}

\section{1. 加强与物流行业企业的合作}

因为在这几年物流行业的发展越来越迅速，所以 高校的物流专业实验室所提供的专业设备也需要进 行更新, 但是因为高校在其中所投入的经费不够充足, 所以难以做到对设备进行及时的更新, 而落后于时代 发展的设备会导致学生对物流行业的认知产生一定 的偏差。因此在经费不充足的情况下，学校可以加强 和物流企业的合作, 满足物流企业的人才需求, 也满 
足学校的教学需求。例如学校可以组织教师或学生定 期或不定期的到物流企业进行学习。教师可以在物流 企业积累更多的实践经验, 而学生也可以在物流企业 提升自己的实践能力, 同时在学生的顶岗实习期间, 学生也可以选择和学校合作的物流企业进行实, 满足 企业对物流专业人才的需求。当然, 学校也可以聘请 企业中的物流专家在学校开展讲座, 给教师和学生传 授更多的经验。

\section{2. 组织专业竞赛}

学校可以开展物流专业竞赛, 在物流专业竞赛中, 学生的学习潜能能够得到有效的激发, 而学生解决问 题的能力也能够得到锻炼, 同时还能够使学生更加了 解物流行业的发展, 完善自己的职业生涯规划。而物 流专业竞赛需要学生们团结合作, 对物流相关知识进 行深入的研究和探讨, 所以在此过程中, 学生的团结 意识能够得到培养, 学生对物流专业的了解也会更加 深入。而学校有必要为优秀的物流专业学生团队给予 一定的奖励, 激发学生的参与热情, 也可以推荐优秀 的学生去参加更高层次的物流专业竞赛, 为学校博得 荣光, 更进一步的锻炼学生的专业能力。

\section{3. 强化科研项目的引导作用}

大部分的科研项目都有其存在的实际意义, 而教 师对物流专业科研项目进行深入的研究是为了推动 物流专业教学的发展, 而教师也可以引导学生完成科 研项目中的子课题, 如果教师能对学生进行良好而正 确的引导, 那么学生就能够在完成课题的过程中得到 很大程度上的提升, 但是如果教师对学生的指导不够 用心, 或是不相信学生的能力, 那么学生在此过程中, 得不到任何的帮助。因此教师要相信学生的能力, 对 学生进行正确的引导, 让学生能够更加顺利的解决问 题。

\section{4. 对智慧物流进行推广的方式}

\section{1. 建设一个完善的智慧物流平台}

针对一些在智慧物流发展中存在的明显问题, 国 家也针对这些问题出台了一些应对对策, 无论是资金 上面的支持还是政策上的鼓励都是希望通过这些措 施能够让一些小型的物流企业能够就当下的智慧物 流进行改进和升级, 从而能够促进智慧物流环节的发 展, 逐渐让物流行业步入智能化、高效化的轨道。通 过对智慧物流行业中的行业资源进行整合, 从而能够 将物流行业的发展成本进行缩减, 达到效益的最大化, 这样就能在最大限度上促进物流行业的发展, 构建一 个高效的物流发展体系。

\section{2. 对智慧物流所需专业人才进行培养}

在互联网时代下对物流行业的发展需要专业的 人才输入, 只有这样才能保持行业的发展创新, 在物 流企业中也是如此，企业之间的发展是否能够得到保 证就在于企业是否具备高素质人才对物流企业发展 起到保障作用, 整个物流行业想要进行智慧物流的稳 步发展就一定要重视对智慧物流专业人才的引进, 通 过对这些拥有专业技术的人才进行引进, 可以带给企 业新的发展角度和发展方向, 为物流行业的发展提供 源源不断的动力, 同时对物流行业的发展变得更加智 能化后消费者的物流体验也会变得更好, 商品之间的 运输也会变得更加高效, 在物流行业方面的人才培养 上也要重视起来, 国家对于此类职业教育也十分重视, 出台了一系列的职业教育方针和支持。在物流企业方 面也要积极引进专业技术人才, 站在时代发展的顶峰 去看待整个行业的发展趋势, 要始终将 “人”作为企 业发展的中心, 对行业的专业人才进行充分合理的安 排和培养, 从而能够给当下物流管理的发展实践带来 帮助。

\section{5. 结论}

智慧物流就是物流行业最新的变化和改革, 但是 智慧物流是否能够得到顺利的前行, 还有待考察, 毕 竟在现阶段的智慧物流应用中, 存在的问题并不少, 物流企业应该加强对智慧物流的完善, 推动智慧物流 的发展就是在推动物流企业的发展, 只有跟随时代的 脚步不断前进，物流企业才能够一直得到市场的青睐。

\section{REFERENCES}

[1] Meng Nan. Embracing the New Era of Internet of Everything -- 2020 World Internet of Things Conference held in Beijing [J]. China Convention and Exhibition (Conference China),2020(24):40-43.

[2] Miao Xingfeng. Construction of Curriculum System for Training Applied Talents at Undergraduate Level of Logistics Management Major [J]. Journal of Guangdong Polytechnic of Light Industry,2020,19(04):48-54.

[3] Sun Xiaoyan, Zhou Yongming, Jiang Wenkai. Research and Practice of Blended Teaching Model of "Smart Logistics" Course [J]. Education and Teaching Forum,2020(53):244-246.

[4] Liu Shu. The Iron and Steel "Smart Port" of Intelligent Drawing and Thing Interconnection -Visiting the Intelligent Logistics Promotion of the Transportation Department of Baosteel [J]. Metallurgical Enterprise Culture,2020(06):55-56.

[5] Li Hang. Logistics Platform Promote the Integration Development of "Manufacturing + Internet + Logistics" [J]. China Market,2020(35):158-159.

[6] Wu Jiangcheng. Research on Synergistic Effect of Logistics Industry and High-tech Industry in 
Shanghai under the Background of Smart Logistics

[J]. Logistics Engineering and Management,2020,42(12):14-16.

[7] Huo Yanfang, Wang Han, Qi Ershi. Building Smart Logistics and Supply Chain, Assisting Intelligent Manufacturing -- Introduction of Smart Logistics and Smart Supply Chain [J]. China Mechanical Engineering,2020,31(23):2891-2897.

[8] Liu Ning. Literature Review on Talent Demand of Intelligent Logistics Technology Professional Group Service Logistics Science and Technology Industry [J]. Time-honored Brand Marketing,2020(12):117118.

[9] Wang Jiansi, Hu Yongquan. "Smart Power" : Research on the Construction of Smart Logistics Strategy of New Manufacturing Enterprises [J]. Modern Business,2020(34):15-17.

[10] Yang Yanhai. Research on the development system and countermeasures of China's smart logistics industry $[\mathrm{J}]$. Technological Economics and Management Research,2020(11):98-102.

[11] "Fourteenth Five-Year Plan" Logistics Industry Development "China Logistics 30 Forum" (4)[N]. Journal of Modern Logistics,2020-11-25(A04).

[12] [12] Zhang Wenhua, Li Fengyan, Weng Shizhou, Li Baimin. Analysis on spatio-temporal distribution difference of A-level logistics enterprises in Guangxi from the perspective of smart logistics $[\mathrm{J}]$. Modern Business Industry,2020,41(35):48-50.

[13] He Fa. Mobile Enabled Intelligent Logistics, CEMAT, a New Member of Slim Family of China Autonomous Robots [J]. Modern Manufacturing,2020(26):24.

[14] Zhou Wangde, Hu Fei. Research on the development strategy of logistics enterprises under the credit system of intelligent logistics public information platform $[\mathrm{J}]$ National circulation economy,2020(31):33-35.

[15] Qian Yingping. Current situation of application of enterprise intelligent logistics technology -- Taking Jingdong Logistics as an example [J]. China Storage and Transportation,2020(11):119-121. 